\title{
Neumonía intersticial usual
}

\author{
Usual interstitial pneumonia
}

\author{
Elena Chevalier Chockee, MD; ${ }^{1}$ Liliana Arias, MD; ${ }^{2}$ Javier Leonardo Galindo, MD; ${ }^{3}$ \\ Jorge Luís Rodríguez, MD; ${ }^{4}$ Carlos Celis-Preciado, MD, MSc. ${ }^{5}$
}

La identificación del patrón de neumonía intersticial usual (NIU) en la tomografía axial computarizada (TAC) de tórax no es sinónimo del diagnóstico de fibrosis pulmonar idiopática (FPI), su adecuada caracterización y la búsqueda de causas secundarias es relevante dado el pronóstico ominoso de la FPI y la existencia de opciones terapéuticas específicas para cada caso (1).

La clasificación actual de la guía de las sociedades ATS/ERS/JRS/ALAT de 2018 define cuatro categorías diagnósticas en la TAC de tórax ante la sospecha de FPI: el patrón de NIU, el patrón probable de NIU, el patrón indeterminado para NIU y el patrón de diagnóstico alternativo (2). El patrón de NIU se define por cuatro características: el panal de abejas, la existencia o no de bronquiectasias o bronquiolectasias de tracción, la distribución de predominio basal y subpleural, y las anormalidades reticulares (Figura 1). Los casos en los que se presenta reticulación periférica con bronquiectasias o bronquiolectasias de tracción, en ausencia del panal de abejas, se definen como un patrón probable de NIU (Figura 2). Los hallazgos de fibrosis que no pueden clasificarse en un patrón definido se categorizan como un patrón indeterminado (Figura 3). En caso de que exista una distribución inusual o hallazgos sugestivos de un patrón correspondiente a otra enfermedad pulmonar fibrosante, se debe plantear un diagnóstico alternativo (Figura 4).

La presencia de panal de abejas tiene un valor predictivo positivo del $90 \%$ $100 \%$ para definir un patrón radiológico de NIU; no obstante, el acuerdo interobservador entre expertos para su identificación es moderado $(\kappa=0,56-0,65)$ $(3,4)$. El panal de abejas corresponde a la presencia de al menos 2 a 3 quistes, agrupados en una o varias capas, de tamaño variable entre 3-10 mm, de paredes gruesas y bien definidas y de localización subpleural (2). El patrón de NIU tomográfico, definido por el hallazgo de panal de abejas, tiene un valor predictivo positivo alto (90\%-100\%) para la identificación de NIU histopatológica $(2,3)$. Sin embargo, se debe anotar que el hallazgo de panal de abejas en la TAC no se relaciona con el hallazgo histopatológico del mismo (5).
${ }^{1}$ Fellow de Neumología, Universidad de Panamá, Hospital Santo Tomás. Ciudad de Panamá, Panamá.

${ }^{2}$ Radióloga, Departamento de Radiología e Imágenes Diagnósticas, Hospital Universitario San Ignacio. Profesora ad honorem Facultad de Medicina, Pontificia Universidad Javeriana. Bogotá D. C., Colombia.

${ }^{3}$ Neumólogo, Unidad de Neumología, Hospital Universitario Mayor. Bogotá D. C., Colombia.

${ }^{4}$ Patólogo, Departamento de Patología, Hospital Universitario San Ignacio. Profesor instructor, Facultad de Medicina, Pontificia Universidad Javeriana. Bogotá D. C., Colombia.

${ }^{5}$ Neumólogo, Unidad de Neumología, Departamento de Medicina Interna, Hospital Universitario San Ignacio. Profesor Asistente, Facultad de Medicina, Pontificia Universidad Javeriana. Bogotá D. C., Colombia.

Autor de correspondencia

Carlos Celis-Preciado. Correo electrónico: celisc@javeriana.edu.co.

Recibido: 8/9/2018. Aceptado: 9/12/2018. 

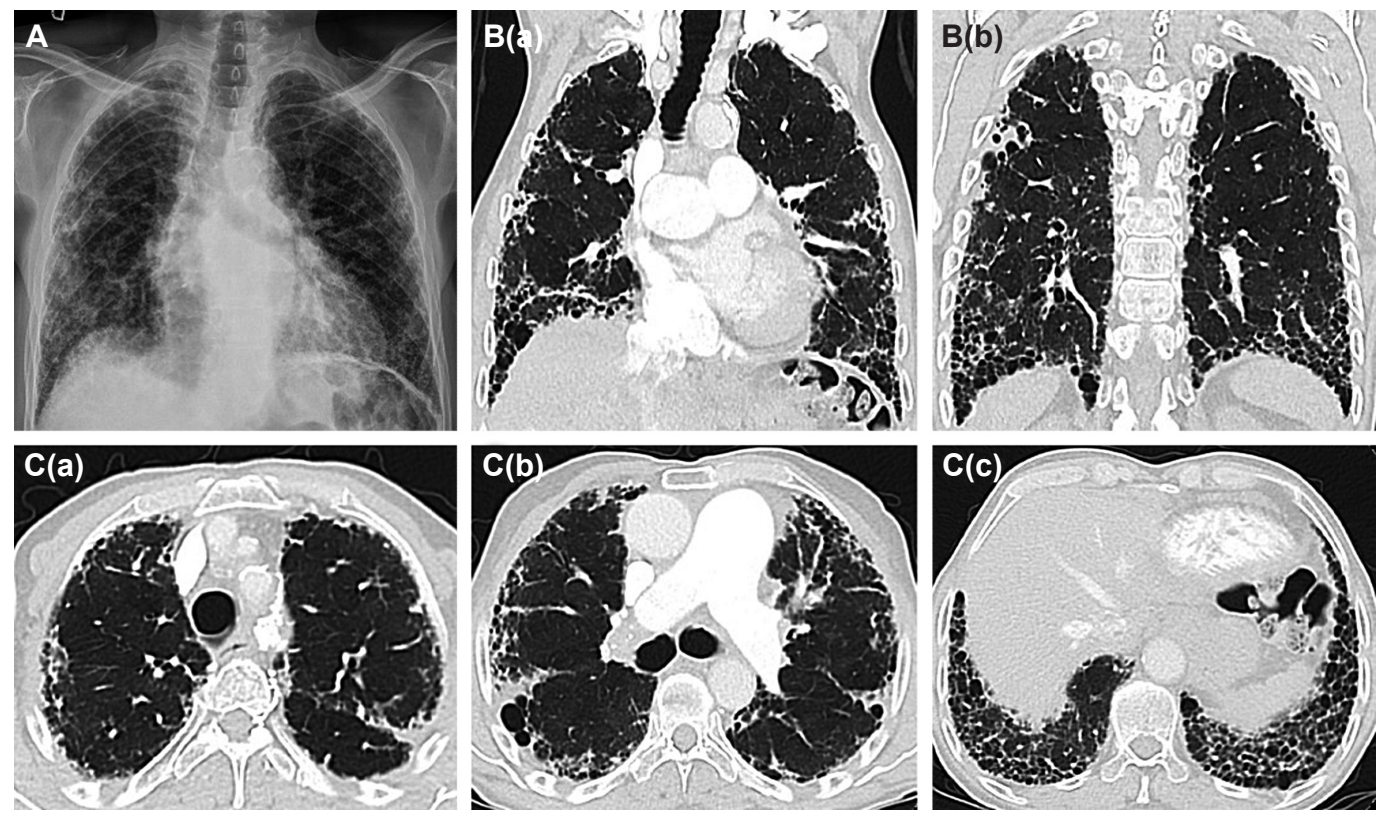

Figura 1. Patrón radiológico de NIU. A. Radiografía proyección posteroanterior: disminución en el tamaño pulmonar, con opacidades reticulares difusas en ambos campos pulmonares y mayor profusión hacia las bases pulmonares. B. Reconstrucciones coronales de TAC: (a) aspecto anterior y (b) aspecto posterior de los hemitórax; estas proyecciones permiten observar con mayor facilidad el gradiente apicobasal de las alteraciones. C. Cortes de TAC: (a) tercio superior, (b) tercio medio y (c) base del tórax; opacidades reticulares subpleurales asociadas con panal de abejas y bronquiectasias de tracción, se observa el gradiente con mayor profusión de las alteraciones hacia las bases pulmonares.
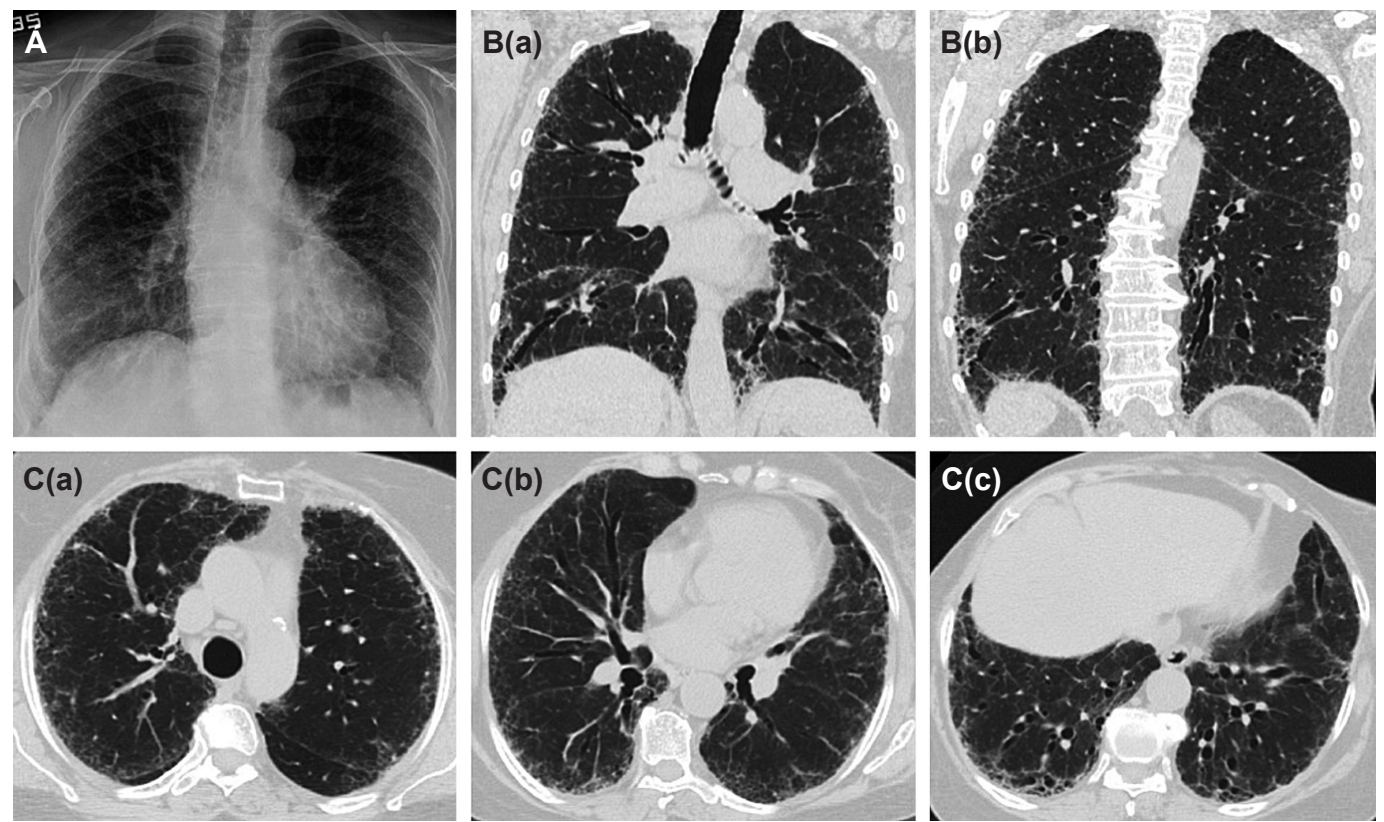

Figura 2. Patrón radiológico de probable NIU. A. Radiografía de proyección posteroanterior: el tamaño pulmonar no se encuentra gravemente comprometido, hay opacidades reticulares difusas. B. Reconstrucciones coronales de TAC: (a) aspecto anterior y (b) aspecto posterior de los hemitórax; respeto de la superficie subpleural en los ápices pulmonares, con opacidades reticulares subpleurales y bronquiectasias de tracción en las bases y aspecto posterior de ambos campos pulmonares. C. Cortes de TAC: (a) tercio superior, (b) tercio medio y (c) base del tórax; opacidades reticulares subpleurales difusas asociadas con bronquiectasias de tracción, las cuales son más evidentes en las bases pulmonares; no se identifica panal de abejas ni hallazgos que sugieran inconsistencia para el diagnóstico de NIU. 

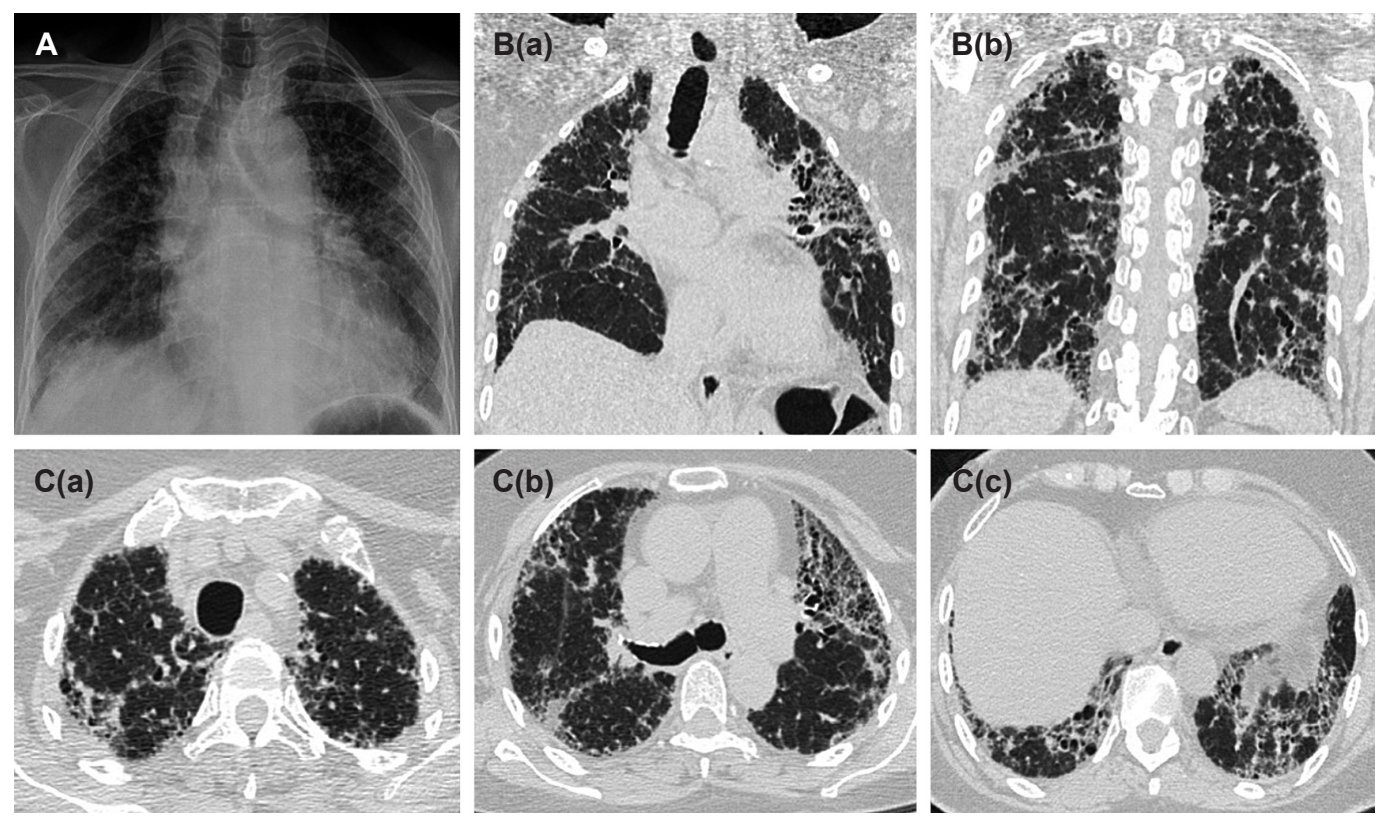

Figura 3. Patrón radiológico indeterminado para NIU. A. Radiografía de proyección posteroanterior: disminución del tamaño de los pulmones, hay opacidades reticulares difusas sin evidencia de gradiente, cardiomegalia y signos de hipertensión pulmonar precapilar. B. Reconstrucciones coronales de TAC: (a) aspecto anterior y (b) aspecto posterior de los hemitórax; estas proyecciones evidencian compromiso difuso anterior y posterior, con asimetría de los cambios, sin establecer un gradiente apicobasal. C. Cortes de TAC: (a) tercio superior, (b) tercio medio y (c) base del tórax; opacidades reticulares difusas subpleurales, asociadas con bronquiectasias de tracción, con asimetría de las alteraciones con mayor profusión en el lóbulo superior izquierdo, panal de abejas en parches y bronquiectasias de tracción. En este caso, las alteraciones se relacionan con un extenso proceso de fibrosis, sin hallazgos que sugieran un diagnóstico probable en una paciente sin antecedentes exposicionales o de enfermedad.
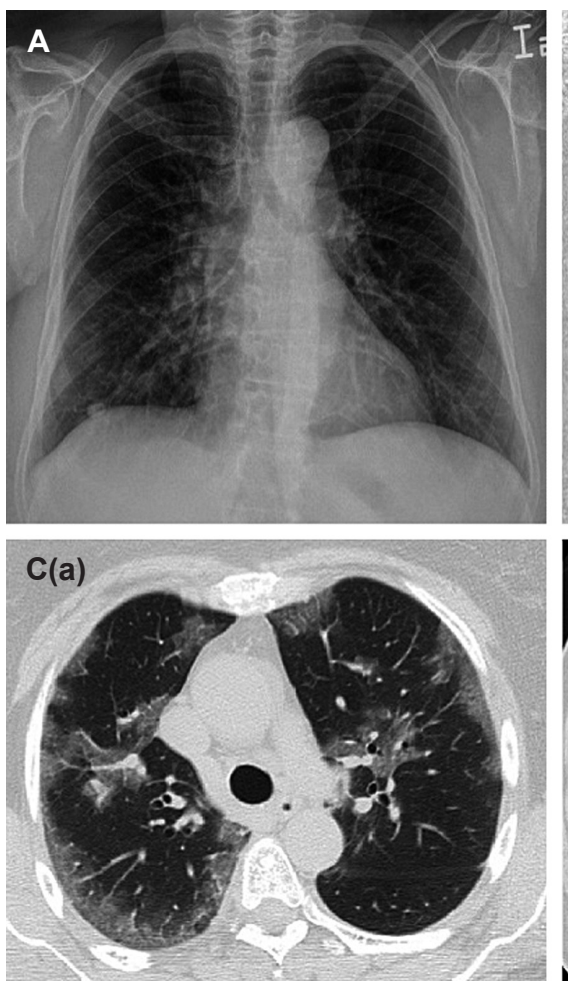
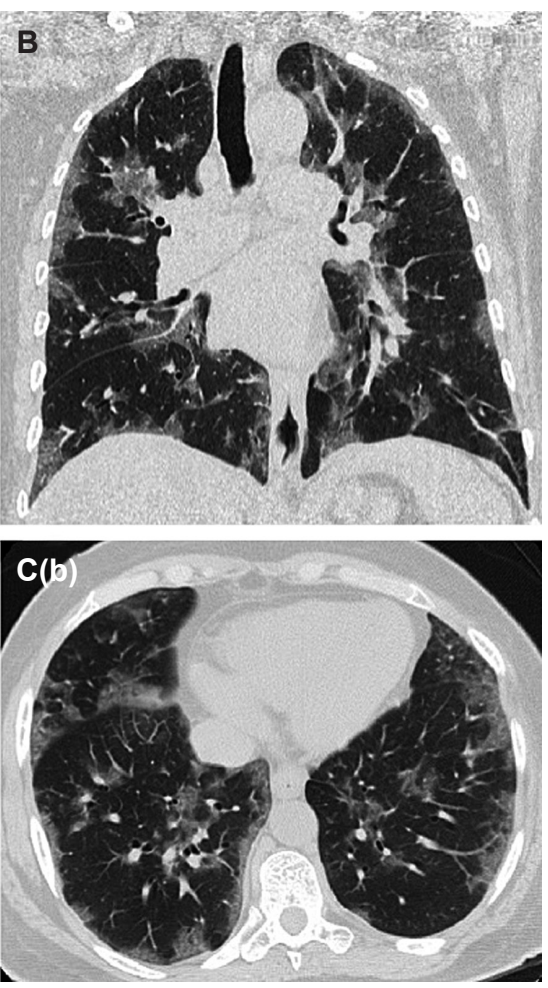

Figura 4. Patrón radiológico consistente con un diagnóstico alternativo a FPI. A. Radiografía de proyección posteroanterior: pulmones de tamaño normal, con un nódulo calcificado en la base pulmonar derecha y signos de hipertensión pulmonar precapilar. B. Reconstrucción coronal de TAC: se identifica una distribución subpleural y broncocéntrica de la enfermedad. C. Cortes de TAC: lóbulos superiores (a) e inferiores (b); parches de vidrio esmerilado de distribución subpleural y broncocéntrica compatibles con un patrón no FPI y con un diagnóstico diferencial más probable correspondiente a un patrón de neumonía de organización, el cual se confirmó por biopsia pulmonar. 
Las bronquiectasias o bronquiolectasias por tracción, en el contexto de un patrón NIU, tienen una localización periférica y pueden coexistir con el panal de abejas (2). Recientemente, se ha sugerido que las bronquiectasias de tracción en la FPI se producen, más que por la tracción mecánica secundaria a la cicatrización, por proliferación displásica bronquiolar secundaria al agotamiento de células madre alveolares; de tal forma que el proceso fisiopatológico de remodelación entre las bronquiectasias de tracción y el panal de abejas es compartido y continuo (6).

Apoyando el valor fisiopatológico del hallazgo de las bronquiectasias por tracción en la FPI, un análisis post hoc de los estudios clínicos INPULSIS mostró que los pacientes con patrón escanográfico de NIU posible, con bronquiectasias por tracción y que no habían sido sometidos a biopsia pulmonar quirúrgica, tenían una progresión clínica y una repuesta al tratamiento con nintedanib similares a las de los sujetos con un patrón de NIU definitivo en la TAC o en la biopsia (7).

El patrón de NIU posible (bajo la clasificación previa de 2011) tiene un valor predictivo positivo de $62 \%$ para la identificación de NIU histopatológica, pero su especificidad y valor predictivo positivo pueden superar el $95 \%$ si se suma a la presencia de una edad mayor de 60 años, de sexo masculino y de un puntaje total de bronquiectasias por tracción $\geq 4$ (establecido por la sumatoria de las bronquiectasias de cada lóbulo: $0=$ ausente, $1=$ leve, $2=$ moderada, $3=$ grave $)(8,9)$. En el caso de que exista el antecedente de tabaquismo y en ausencia de otras posibles causas de fibrosis pulmonar, el diagnóstico de FPI podría establecerse con confianza (10).

La Sociedad de Fleischner propuso un cambio en los criterios radiológicos de la FPI, dándole mayor peso al hallazgo de las bronquiectasias o bronquiolectasias de tracción para el diagnóstico de la enfermedad, lo que sugiere que podría definirse la FPI con confianza en presencia de un patrón probable de NIU, en un contexto clínico adecuado en el que se descarte la existencia de causas secundarias, sin requerimiento de biopsia quirúrgica (Tabla 1) (10). La actual guía de diagnóstico de la FPI de las sociedades ATS/ERS/JRS/ ALAT acogió parcialmente los cambios, pero sugiere que los pacientes con patrón probable de NIU sean lle-

Tabla 1. Patrones radiológicos de NIU $(2,10)$

\begin{tabular}{|c|c|c|}
\hline Patrones & Hallazgos & Distribución \\
\hline NIU & $\begin{array}{l}\text { - Panal de abejas. } \\
\text { - Bronquiectasias o bronquiolectasias periféricas por tracción. } \\
\text { - Ausencia de hallazgos que sugieran diagnóstico alternativo. }\end{array}$ & $\begin{array}{l}\text { Predominante basal y } \\
\text { subpleural; con frecuencia } \\
\text { es heterogénea. }\end{array}$ \\
\hline Probable NIU & $\begin{array}{l}\text { - Patrón reticular con bronquiectasias o bronquiectasias periféricas por tracción. } \\
\text { - Panal de abejas ausente. } \\
\text { - Ausencia de hallazgos que sugieran diagnóstico alternativo. Puede haber } \\
\text { leve vidrio esmerilado sobre las áreas de reticulación. }\end{array}$ & $\begin{array}{l}\text { Predominante basal y } \\
\text { subpleural; con frecuencia } \\
\text { es heterogénea. }\end{array}$ \\
\hline $\begin{array}{l}\text { Indeterminado } \\
\text { para NIU }\end{array}$ & $\begin{array}{l}\text { - Patrón reticular sutil. Puede haber distorsión o vidrio esmerilado leve. } \\
\text { - Evidencia de fibrosis que no sugiere una etiología específica. }\end{array}$ & $\begin{array}{l}\text { Predominante basal y } \\
\text { subpleural. }\end{array}$ \\
\hline $\begin{array}{l}\text { Hallazgos } \\
\text { consistentes } \\
\text { con un } \\
\text { diagnóstico } \\
\text { alternativo }\end{array}$ & $\begin{array}{l}\text { Hallazgos sugestivos de otro diagnóstico: } \\
\text { - Predominante consolidación. } \\
\text { - Opacidad en vidrio esmerilado pura extensa (sin exacerbación). } \\
\text { - Mosaico de atenuación extenso. } \\
\text { - Nódulos o quistes difusos. } \\
\text { - Placas pleurales (considere la asbestosis). } \\
\text { - Esófago dilatado (considere enfermedad del tejido conectivo). } \\
\text { - Erosiones claviculares distales (considere artritis reumatoide). } \\
\text { - Adenomegalias (evalúe otras etiologías). } \\
\text { - Derrames pleurales o engrosamiento pleural (considere enfermedad del } \\
\quad \text { tejido conectivo o uso de medicamentos). }\end{array}$ & $\begin{array}{l}\text { - Fibrosis predominante en } \\
\text { zona superior o media. } \\
\text { - Predominante } \\
\text { peribroncovascular o } \\
\text { perilinfático con respeto } \\
\text { subpleural. }\end{array}$ \\
\hline
\end{tabular}


vados a una prueba complementaria, previa valoración de la probabilidad de NIU, ya sea con la evaluación de la citología del lavado bronquioalveolar o con la toma de biopsia pulmonar $(2,11)$.

\section{Conflictos de interés}

Los autores no declaran conflictos de interés con el presente artículo.

\section{Referencias}

1. Martinez FJ, Chisholm A, Collard HR, Flaherty KR, Myers J, Raghu G, et al. The diagnosis of idiopathic pulmonary fibrosis: current and future approaches. Lancet Respir Med. 2017;5(1):61-71.

2. Raghu G, Remy-Jardin M, Myers JL, Richeldi L, Ryerson CJ, Lederer DJ, et al. Diagnosis of idiopathic pulmonary fibrosis. An official ATS/ERS/JRS/ALAT clinical practice guideline. Am J Respir Crit Care Med. 2018;198(5):e44-e68.

3. Raghu G, Collard HR, Egan JJ, Martinez FJ, Behr J, Brown KK, et al. An official ATS/ERS/JRS/ALAT statement: idiopathic pulmonary fibrosis: evidence-based guidelines for diagnosis and management. Am J Respir Crit Care Med. 2011;183(6):788-824.

4. Walsh SL, Calandriello L, Sverzellati N, Wells AU, Hansell DM; UIP Observer Consort. Interobserver agreement for the ATS/ERS/JRS/ALAT criteria for a UIP pattern on CT. Thorax. 2016;71(1):45-51.
5. Chung JH, Chawla A, Peljto AL, Cool CD, Groshong SD, Talbert JL, et al. CT scan findings of probable usual interstitial pneumonitis have a high predictive value for histologic usual interstitial pneumonitis. Chest. 2015;147(2):450-9.

6. Piciucchi S, Tomassetti S, Ravaglia C, Gurioli C, Gurioli C, Dubini A, et al. From "traction bronchiectasis" to honeycombing in idiopathic pulmonary fibrosis: A spectrum of bronchiolar remodeling also in radiology? BMC Pulm Med. 2016;16(1):87.

7. Raghu G, Wells AU, Nicholson AG, Richeldi L, Flaherty KR, Le Maulf F, et al. Effect of nintedanib in subgroups of idiopathic pulmonary fibrosis by diagnostic criteria. Am J Respir Crit Care Med. 2017;195(1):78-85.

8. Brownell R, Moua T, Henry TS, Elicker BM, White D, Vittinghoff $\mathrm{E}$, et al. The use of pretest probability increases the value of high-resolution $\mathrm{CT}$ in diagnosing usual interstitial pneumonia. Thorax. 2017;72(5):424-9.

9. Homer R, Lederer DJ. Diagnosing idiopathic pulmonary fibrosis without a lung biopsy: honeycombing not required. Thorax. 2017;72(5):391-2.

10. Lynch DA, Sverzellati N, Travis WD, Brown KK, Colby TV, Galvin JR, et al. Diagnostic criteria for idiopathic pulmonary fibrosis: a Fleischner Society White Paper. Lancet Respir Med. 2018;6(2):138-53.

11. Richeldi L, Wilson KC, Raghu G. Diagnosing idiopathic pulmonary fibrosis in 2018: bridging recommendations made by experts serving different societies. Eur Respir J. 2018;52(3). pii: 1801485 . 